\title{
The mass ratio and initial mass functions in spectroscopic binaries
}

\author{
J. R. Ducati ${ }^{1}$, E. M. Penteado ${ }^{1,2, \star}$, and R. Turcati ${ }^{1, \star \star}$ \\ 1 Departamento de Astronomia, Universidade Federal do Rio Grande do Sul, Av. Bento Gonçalves 9500, 91 501-970 Porto Alegre, \\ Brazil \\ e-mail: ducati@if.ufrgs.br \\ 2 Observatório do Valongo, Universidade Federal do Rio de Janeiro, Ladeira Pedro Antônio 43, 20 080-090 Rio de Janeiro, Brazil
}

Received 17 December 2009 / Accepted 8 September 2010

\begin{abstract}
Context. Spectroscopic binaries are important in studies of binary star-formation, since original information linked to the forming stars, such as the mass ratio, can be kept in the system and observationally retrieved.

Aims. We aim to derive the more probable mass ratio distributions, to test a possible Initial Mass Functions acting on the formation of spectroscopic binaries, and to derive the value of $q_{0}$, the minimal mass ratio for which both binary components are simultaneously in the main sequence.

Methods. The sample has 249 systems, selected from the Ninth Catalogue of Spectroscopic Binaries, with selection criteria that include only those systems with periods longer than four days and magnitudes brighter than $m_{V}=7.0$, and main-sequence primaries, to avoid mass exchange. Monte Carlo simulations of observational parameters were performed. We performed simulations by comparing an observational quantity, $Y_{\mathrm{obs}}$, equal to $f(m) / m_{1}$, where $f(m)$ is the mass function, to theoretical $Y_{\text {th }}$, which is a function of the system inclination (assumed to be random), and of the mass ratio $q=m_{2} / m_{1}$. We considered a minimal mass ratio $q_{0}$, which is the limiting condition, to ensure that both components are simultaneously on main sequence, avoiding evolutionary effects leading to mass exchange and loss of information on the original masses. Calculations were done using several $q_{0}$ values. The form of the distribution of mass ratios was studied by simulations of $Y$, involving several functions, with slopes which are increasing, decreasing, constant, or bimodal. Other simulations were done by the generation of stars following several initial mass function laws. We combined pairs of stars generated from different IMFs, assuming that binaries arise from the pairing of stars simultaneously formed. In this case, several values of $q_{0}$ were also used in the input equations.

Results. Our results indicate that decreasing $q$ distributions match better with observations and also that a unique IMF gives better fits to observations than a composite IMF, with a slope around 1.4, rather than the widely used 2.35 slope. Values of $q_{0}$ tend to be lower than what is suggested by previous studies, which point to $q_{0}$ around 0.2 ; all simulations suggest that $q_{0}$ values as low as 0.08 produce the best fits to observations.
\end{abstract}

Key words. binaries: spectroscopic

\section{Introduction}

The understanding of star formation can benefit from data of binary stars, since these systems carry information which is lost when single stars form (Larson 2001). However, formation processes of binary and multiple stars are far from being completely understood. Possible processes include simultaneous condensation from a common primordial nebula, fission, and aftercondensation capture. Numerical simulations involving now available high-performance computing have provided some new insights on this question (Tohline \& Durisen 2001). Alternative approaches can use models describing those processes taking as a fundamental parameter the mass ratio, $q=m_{2} / m_{1}\left(m_{1}\right.$ and $m_{2}$ being the primary and second masses of the binary components), on the grounds that some values of $q$ are only possible from certain processes, and that different physical processes can favor higher or lower values of the mass ratio. The form of the mass ratio distribution, $f(q)$, has been the object of extensive

* Present address: Greenberg fellow, Leiden Observatory, University of Leiden, PO Box 9513, 2300 RA Leiden, The Netherlands

e-mail: penteado@strw.leidenuniv.nl

$\star \star$ Present address: Centro Brasileiro de Pesquisas Físicas, Rua Dr. Xavier Sigaud 150, 22 290-180 Rio de Janeiro, Brazil

e-mail: turcati@cbpf.br discussion on whether $f(q)$ decreases or increases with increasing $q$, if it has a bimodal behavior, or if $f(q)$ depends on the orbital period. Model-fitting approaches have produced results in every direction. Decreasing functions, of the form $q^{-7 / 3}$, were suggested by Jaschek \& Ferrer (1972); this function has some theoretical background, because it is the original mass function of Salpeter (1955), as pointed out by Jaschek (1969). Besides, this paper by Jaschek and Ferrer introduced the parameter $q_{0}$, the minimal mass ratio that ensures that both stars are simultaneously at the main sequence, thus avoiding conditions where evolutionary processes lead to mass exchange and loss of information on primordial component masses. Concerning mass ratio distributions, similar results were published more recently by Heacox (1998). However, more results have been published favoring other $f(q)$ 's. Hogeveen $(1990,1992 \mathrm{a}, \mathrm{b})$ finds that $f(q)$ can be a power law, either decreasing or increasing, depending on whether the sample (from Batten et al. 1978 Eighth Catalogue of Spectroscopic Binaries - SB) was formed by single-line (SBI) or double-line (SBII) systems. This calls attention to the extent to which selection effects can influence results. Increasing $f(q)$ s, with peaks near high $q$ 's were suggested by Lucy \& Ricco (1979), among others, for close SBs; however, most studies with similar results (Garmany et al. 1980; Abt \& Levy 1985; Levato et al. 1987; Fekel et al. 1988) are based on samples composed 
of close systems, where contact or mass exchange lead to loss of information on the original mass of the components. Bimodal distributions have been suggested by Trimble (1974), and as well by Abt \& Levy (1976); finally, both $q^{n}$ and bimodal distributions were the results of model-fittings by Dabrowski \& Beardsley (1977) and Trimble \& Ostriker (1978). Also based on Batten's Eighth Catalogue is the study by Fisher et al. (2005) concerning a sample of local field stars, which indicated a $q$ distribution of field binaries with many $q$ values near unity, and thus dominated by double-lined systems. The above results indicate that confusion does exist, a problem that can be approached by the use of carefully selected samples where biases are minimized. The recent release of a large database on spectroscopic binary stars has allowed more significant model-fitting tests. The choice of spectroscopic systems is convenient because of the availability of useful parameters, especially radial velocities, which in visual binaries lack the necessary accuracy and amplitude. Using a selected sample of spectroscopic binaries as a reference, the scope of this work is threefold: to look for the more probable mass ratio distributions, to test Initial Mass Functions acting during binary formation, and to derive values for the parameter $q_{0}$; to reach these objectives, we performed Monte Carlo simulations involving assumptions on mass ratio distributions, on initial mass functions, and testing several $q_{0}$ values.

\section{Data and basic methodology}

Following former compilations of spectroscopic binary observational parameters, for example, that by Batten et al. (1978), we selected the observational sample from the online version of the Ninth Catalogue of Spectroscopic Binary Orbits (Pourbaix et al. 2004), which provides information on 2385 systems. Observational biases are certainly present, and several criteria were applied to compile a sample with a bias as small as possible, as follows:

a) An important bias in the Ninth Catalogue is that the percentage of eclipsing binaries is statistically increased. This happens because variability is easily detected, and systems with deeper eclipses are even more easily discovered, because they have components of similar brightness and, therefore, with higher mass ratios. This bias in favor of eclipsing $\mathrm{SBs}$ is stronger in fainter systems. To minimize this bias, the sample included only binaries brighter than $m_{V}=7.0$, on the grounds that those objects belonging to the bright star catalogue (BSC) and its Supplement (Warren \& Hoffleit 1987), which list systems brighter than $m_{V}=7.0$, have been extensively observed and few spectroscopic systems, if any, remain undetected.

b) Mass exchange may influence the evolution of close systems, so to ensure a sample that conserves the original information of both components, the sample included only systems with periods longer than four days, corresponding to orbits with semi-major axis of about 7.4 million kilometers. This value is about $40 \%$ higher than the largest $\mathrm{O}$ stars in the sample, and accordingly, systems with possible contact between the components were not included in the compilation. This criterion leads to the exclusion of certain pairs of multiple systems (HD 25 204, HD 36 695, HD 71663 , HD 157 482, HD 201433 ), even if these systems would have remained in the sample due to other criteria.

c) Another condition imposed to avoid mass exchange was the exclusion of all systems with non-main sequence primaries. These last two criteria are complementary, producing a sample with non-interacting stars even for the eventual systems which, being formed by capture, do not have coeval components.

The final, homogeneous sample, had 249 systems, with primary spectral types distributed as follows: $29 \%$ OB, 55\% AF, $16 \%$ GK. The list of stars is given in Table 2 at the end of this paper. This sample is significantly greater than those used in most of the papers cited in Sect. 1, and includes single (144 objects) and double-lined (105) systems.

A special mention has to be made concerning the paper by Fisher et al. (2005), because this paper, in a first analysis, has partially similar scopes and sources of data. However, an important objective for us was to derive values of $q_{0}$, a parameter which was not approached in their paper because it is seldom studied. Approaches to the other scopes are heavily influenced by the sample used, and here, it is true that the present sample of 249 systems is smaller than that of Fisher and collaborators, which has 371 objects; these samples, however, have some important differences. The larger sample was compiled with data from Batten's Eighth Catalogue and from private communications; it used all values of periods, thus allowing the inclusion of contact systems and evolved stars, where mass exchanges can occur, processes which modify the original mas ratios at system formation. Unfortunately, the paper by Fisher et al. (2005) does not provide the list of systems forming the sample used for their study, so a direct comparison is not possible. However, from their criteria of $d \leq 100 \mathrm{pc}$ and $M_{V} \leq 4$, it can be deduced that for later stars, $\mathrm{G}$ and $\mathrm{K}$ dwarfs are excluded, because of the luminosity criterion. On the earlier side, an examination of the Bright Star Catalogue suggests that there are significant differences between Fisher's and our sample. For example, from the BSC it is evident that the criterion for distances smaller than $100 \mathrm{pc}$ only allows the inclusion, in Fisher's sample, of about eight dwarfs of spectral type B3 or hotter, not necessarily primaries of spectroscopic binaries. On the other hand, GK dwarfs make up 16\% of primaries of our sample, which besides includes $31 \mathrm{~B} 3$, or earlier, dwarf primaries. The same reasoning extends to all $\mathrm{B}$ dwarfs. Consequently, even if the exact content of Fisher's list is not known, a clear perception arises that the ensemble of these differences is very significant and results in very different samples, for the spectral types and luminosity classes, and also for the volume of space studied.

Mass ratios can be directly derived from the radial velocities measured from SBIIs; however, with the present number of 105 systems, they are not enough to provide reliable information about the form of a mass ratio distribution. Besides, in doing this, the resulting sample presents a bias towards higher mass ratios, a perception already expressed at the modelings performed by Tout (1991). This author, and also Hogeveen (1992a), use different arguments to show that an inverse bias is present if only SBI systems are used. Indeed, both cite and use a former result, summarized in Fig. 1 of Staniucha (1979). The database used to produce this result carries the biases which are avoided in our sample. Also, given a fixed sample, the relative proportion of SBI to SBII will change steadily in favor of SBII, as time passes and observational techniques improve. Modeling this shifting subsamples separately would give unreliable results. So, the question is how to avoid those biases, and at the same time, how to put the observational information from systems with only one spectrum into a useful form. The so-called "mass function", $f(m)$, is a well known expression that addresses this problem. Not to be confused with the Initial Mass Function, the $f(m)$ for spectroscopic binaries is derived from Kepler's third 
J. R. Ducati et al.: The mass ratio and initial mass functions in spectroscopic binaries

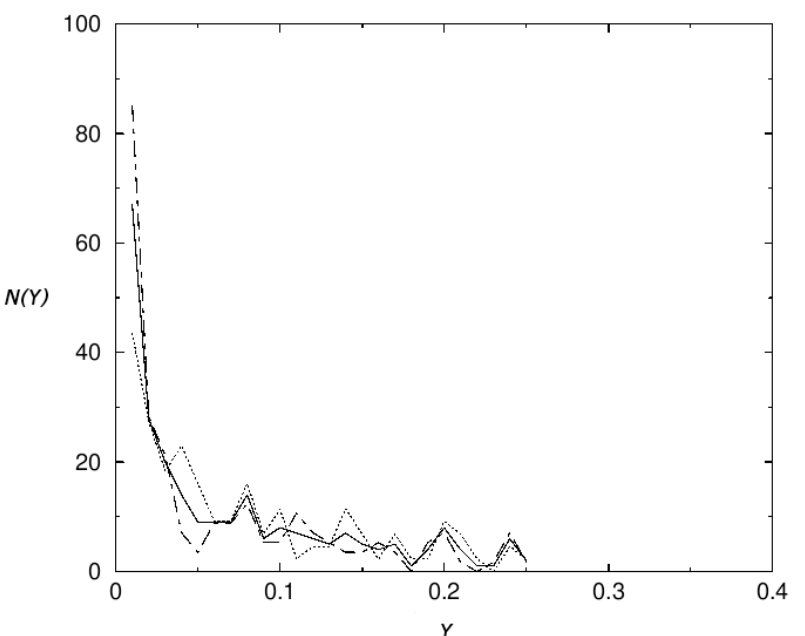

Fig. 1. Histogram for observed $Y$, from 249 spectroscopic binaries (solid line). Also shown are normalized tracings of sub-samples, for periods longer (dotted line) or shorter (dot-dashed line) than 40 days.

law, and has been presented and used in Halbwachs (1987) or in Duquennoy \& Mayor (1991) among others. It is given by

$f(m)=\frac{m_{2}^{3} \sin ^{3} i}{\left(m_{1}+m_{2}\right)^{2}}$

with $m_{1}$ and $m_{2}$ defined as above, and $i$ the inclination of the plane of the system with respect to the line of sight. The mass function can also be expressed in terms of observational quantities, in the form

$f(m)=1.0385 \times 10^{-7} k_{1}^{3} P\left(1-e^{2}\right)^{3 / 2}$

where $k_{1}$ is the radial velocity of the primary component, in $\mathrm{km} \mathrm{s}^{-1}, P$ is the period in days, and $e$ is the system's orbital eccentricity. This allows the use of SBI in exactly the same way as for SBII, as was pointed by Trimbe (1990), with the advantage that only information from the primary is needed. In a lengthy analysis, and after stating that it is impossible to determine an overall mass-ratio distribution by adding the observed $q$ distributions of SBI and SBII systems, Hogeveen (1992b) finally suggests that the observed $q$ distribution of SBII systems is compatible with the assumption that all binary stars in the solar neighborhood have mass ratios distributed according to the intrinsic $q$ distribution found from SBI systems. The purpose of this study is to perform a comparison of observational information from Eq. (2) with theoretical predictions from Eq. (1); here, a problem arises because to use Eq. (1) one has to know $m_{2}$, a piece of information that is absent from Eq. (2). This difficulty can be negotiated by dividing both Eqs. (1) and (2) by $m_{1}$. This gives, for the theoretical expression,

$\frac{f(m)}{m_{1}}=\frac{q^{3} \sin ^{3} i}{(1+q)^{2}} \equiv Y_{\mathrm{th}}$

and for the observational equation

$\frac{f(m)}{m_{1}}=\frac{1.0385 \times 10^{-7} k_{1}^{3} P\left(1-e^{2}\right)^{3 / 2}}{m_{1}} \equiv Y_{\mathrm{obs}}$.

The modeling of $Y_{\text {th }}$, as just defined, will be compared with observations, expressed by $Y_{\mathrm{obs}}$. To obtain $Y_{\mathrm{obs}}$, the masses of the primary stars are needed. With the spectral types (main sequence) of the primaries being known for all 249 objects in the
Table 1. List of mass ratio, $q$ distributions used in the construction of histograms for theoretical $Y$.

\begin{tabular}{ccc}
\hline \hline Number & $f(q)$ & Function is \\
\hline 01 & constant & constant \\
02 & $q$ & increasing \\
03 & $q-q_{0}$ & increasing \\
04 & $1-q$ & decreasing \\
05 & $1-a q$ & decreasing \\
06 & $q^{-2}$ & decreasing \\
07 & $q^{-\frac{7}{3}}$ & decreasing \\
08 & $\left(q-q_{0}\right)^{2}$ & bimodal \\
09 & {$\left[q-\frac{\left(1+q_{0}\right)}{2}\right]^{2}$} & bimodal \\
10 & $q^{\frac{1}{3}}$ & increasing \\
11 & $\left(q-q_{0}\right)^{\frac{1}{3}}$ & increasing \\
\hline
\end{tabular}

sample, the masses of the primaries can be obtained from a spectral type-mass table, like that from Lang (1991), where missing spectral types had their masses determined by iteration. With data on periods and eccentricity from the Ninth Catalogue, it was possible to derive the mass function, and $Y_{\mathrm{obs}}$. The histogram for $Y_{\text {obs }}$ is shown in Fig. 1. To investigate a possible dependency of the period on the mass function, the observational sample was divided into two groups: periods longer or shorter than 40 days, a value that approximately divides the sample into two equal parts. The normalized traces for these sub-samples are also shown in Fig. 1.

\section{Simulations from mass ratio functions}

The function $Y_{\text {th }}$ depends on the form of the distribution function of mass ratios, $f(q)$. The modeling of $Y_{\mathrm{th}}$, which will be compared to $Y_{\mathrm{obs}}$, thus totally depends on the expression of $f(q)$. This is done by assuming several test $f(q)$ 's, which are each used to generate a $Y_{\mathrm{th}}$. With respect to the $\sin i$ dependency in Eq. (3), inclinations of systems are supposed to be random, as widely accepted (Halbwachs 1987). The choice of test $f(q)$ 's must cover most of the possible slopes or forms. Table 1 lists the $f(q)$ 's used in simulations.

The construction of the histograms for $Y_{\text {th }}$ was made for each $q$ distribution listed in Table 1 , by generation of successive $Y_{\text {th }}$ values using random numbers. The procedure is as follows: given any distribution $f(q)$,

$\frac{\int_{q_{0}}^{q} f(q) \mathrm{d} q}{\int_{q_{0}}^{1} f(q) \mathrm{d} q}=x$.

Here, $x$ is a random number and the $q_{0}$ parameter is introduced, which is the minimal mass ratio that ensures that both stars are simultaneously at the main sequence, thus avoiding conditions where evolutionary processes lead to mass exchange and loss of information on primordial component masses; in this sense, $q_{0}$ is a critical parameter for this study, and finding its value is a result in itself. It can be determined from evolutionary lifetime models, following Iben (1964). A system in which the primary is too massive with respect to its secondary will never have both components simultaneously in the main sequence, because by the time the secondary arrives at the ZAMS, the primary will already have left it. The approximate limit of $q_{0}$ for coexistence as dwarfs would be around 0.17 , as suggested by Giannone \& Giannuzzi (1969) and Jaschek (1969). Solving the integrals (Eq. (5)) for each distribution leads to an expression for $q$ that 


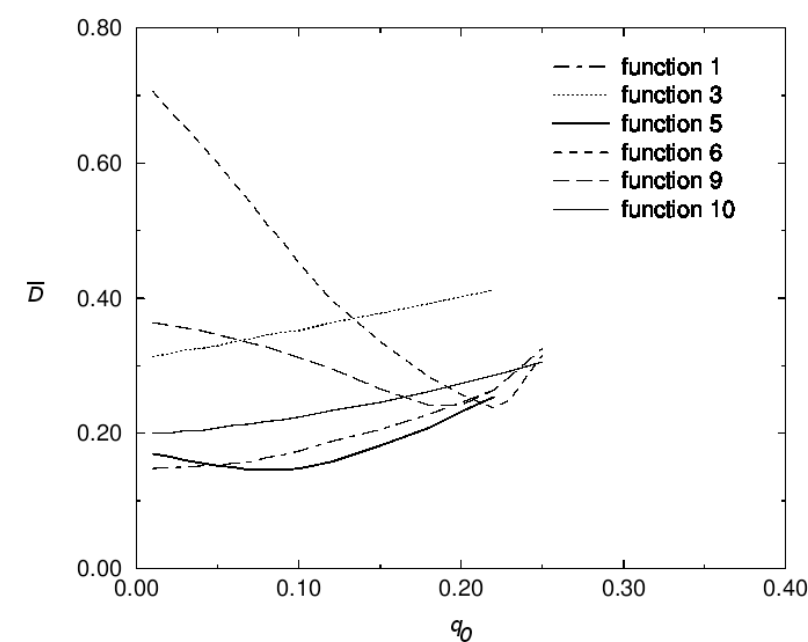

Fig. 2. Behavior of simulations of binary formation, compared to observations. Several mass distributions from Table 1 are shown. Distributions favoring lower mass ratios and values of $q_{0}$, the minimal mass ratio around 0.08 provides the best fits.

contains both $q_{0}$ and $x$. A similar procedure is performed for the sine component. Values of modeled $Y_{\text {th }}$ (Eq. (3)) are generated in this way and successive executions lead to the construction of histograms, one for each adopted $f(q)$ distribution, where a $q_{0}$ value is adopted. These modeled histograms have to be compared to the $Y_{\mathrm{obs}}$ histogram. The comparison takes into account that the observational sample contains non-negligible noise, because it is relatively small ( 249 objects), even if it is one of the largest compiled to date. So, simulations for each $f(q)$ were, accordingly, generated up to 249 executions, producing histograms of $Y_{\text {th }}$ which have the same size as the $Y_{\text {obs }}$ histogram; the histogram for $Y_{\text {th }}$ has a noise similar to the $Y_{\text {obs }}$ noise and, because it has the same size, allows also a comparison on a normalized basis. Both distributions, $Y_{\mathrm{obs}}$ and $Y_{\mathrm{th}}$, are compared point-to-point for 25 partitions in the domain of possible $Y$ values, which go from 0 to 0.25 . The modulus of the difference within any partition $k$ is

$\operatorname{dif}_{k}=\left|Y_{\mathrm{obs}, k}-Y_{\mathrm{th}, k}\right|$

and the total difference between the histograms is

$D=\sum_{k=1}^{25} d i f_{k}$.

This comparison is performed repeatedly to generate a mean difference, which is given by

$\bar{D}=\sum_{j=1}^{N} D_{j}$.

Here, $N$ was set to 1000 , a value sufficient for the convergence of $\bar{D}$. This procedure, which has made on a normalized basis, produces values of $\mathrm{D}$ within the interval $(0,1)$, where $\bar{D}=1$ means a model totally different from the observations. Values of $D$ for selected test distributions from Table 1 are presented in Fig. 2, which also gives information on the minimal mass ratio, $q_{0}$.

\section{Simulations from initial mass functions}

Another modeling of the observed $Y$ was performed by creating binary systems from the random combination, two by two, of stars generated following a formation law: the Initial Mass Function. The expression of the IMF, since its first formulation by Salpeter (1955), has been frequently modified. More recently, Kennicut (1998) suggested that observations are best fitted by a composite IMF, with a slope 1.4 for formation up to one solar mass, and a slope 2.35 for greater masses. However, the situation can be different when star-forming cores undergo fragmentation, a scenario studied by Goodwin \& Kroupa (2005). In these cases, a binary can be formed after additional members are ejected from the system, leading to the formation of close binaries. This would fit the description of many spectroscopic binaries, in which case a slightly different IMF could apply. The fragmentation process can produce stars of different masses, the smaller ones are the first to be ejected. The two remaining masses may or may not obey a specific, even composite IMF. To test these possibilities we performed a simulation of the random combination of stars that are independently formed. Here, the variables are the minimum and maximum masses possible in the star-formation processes and the IMF slopes; many values around those suggested by Kennicut were tested. The minimal mass was set to 0.06 solar masses; the upper limit for stellar masses was set to 120 solar masses, a conservative approach to the value suggested by Figer (2005). The generation of masses $m_{1}$ and $m_{2}$ starts with the equation

$$
\frac{\int_{m_{\min }}^{m} m^{-t} \mathrm{~d} m}{\int_{m_{\min }}^{m_{\max }} m^{-t} \mathrm{~d} m}=x
$$

where $t$, the slope of the IMF is also a variable with values $t_{1}$ and $t_{2}$, allowing the existence of a composite IMF (Kennicut 1998). The generation of $m_{1}$ and $m_{2}$ is subject to conditions that, if $t$ is $t_{1}$, masses greater than a critical value (here, one solar mass) are discarded; and if $t$ is $t_{2}$, masses smaller than the critical value are discarded. Here, $t_{1}$ and $t_{2}$ are first defined; which one will be used comes from a random sorting; this $t_{i}$ value is then used to generate $m_{1}$, and after a new $t_{i}$ definition, the mass $m_{2}$ is generated. Therefore, in all systems created by this simulation, IMFs for primaries and secondaries can be different. The same consideration for $q_{0}$ already made in Sect. 3 applies. Repeated executions are performed, and applied in Eq. (3) together with the random $\sin i$, to produce a histogram of simulated $Y_{\text {th }}$, which can be compared to the observations. We did this following the same procedure described in the previous section; the number of modules of point-to-point subtractions, observational minus modeling, was also set to be 1000 . We show some results for various slopes and $q_{0}$ in Fig. 3.

\section{Discussion and concluding remarks}

An inspection of Fig. 1 does not provide evidence of a period dependency on the $Y$ distribution and, therefore, the form of $f(q)$ does not seems to be period-dependent. An analogous histogram is found in Fisher et al. (2005), with a different shape; indeed, since the criteria used to compile the samples are very different, a similarity is not expected. The question of the form of the mass ratio distribution in spectroscopic binaries as well as on the $q_{0}$ value can be addressed by an analysis of Fig. 2, where the histogram of the entire sample of 249 stars was compared with the various simulations; similar calculations using the subsamples for shorter and longer periods did not produce significantly different results. We analysed the evolution of $D$ values because several $q_{0}$ are used in each $q$ distribution. From Fig. 2 it is clear that those $q$ distributions that are bimodal or 
J. R. Ducati et al.: The mass ratio and initial mass functions in spectroscopic binaries

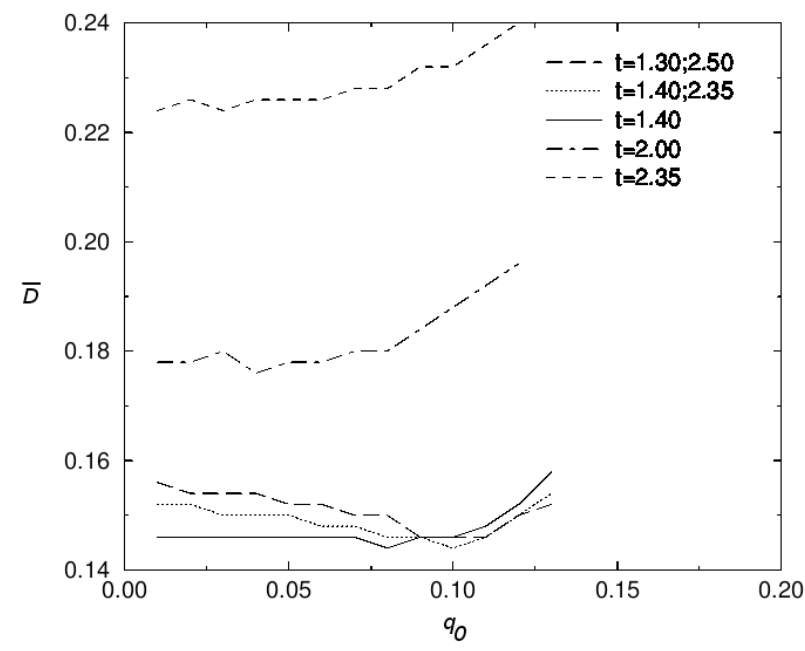

Fig. 3. Behavior of simulations of binary formation, compared to observations. Several slopes (the " $t$ " parameter) of initial mass functions are shown. The IMFs with smaller slopes, together with $0.06 \leq q_{0} \leq 0.1$, provide the best fits.

increase with $q$, have higher $D$, regardless of which $q_{0}$ is used. Therefore, this family of functions is unsuitable for the present simulations. Another group of functions seems to provide better fits. A mass ratio distribution with uniform probability (function 1) gives good results. However, linear, decreasing $q$ functions in the form $f(q)=1-a q$ with $a \approx 0.5$, produce the best fits to observations, and also give important information on the $q_{0}$ value, which is around 0.08 . These functions are preferred over function 1 because they provide information on the constraint on $q_{0}$, while function 1 only returns low $\mathrm{D}$ values for unphysical (i.e. too low) values of $q_{0}$. Function 5 with $a=0.5$ seems to be the best compromise between low $D$ and a physical $q_{0}$. Consequently estimates of $q_{0}$ around 0.2 do not produce the best results. A $q_{0}$ from 0.08 to 0.1 , meaning that one component can be more than ten times more massive than the other, puts limits on the primary masses, giving preference to the formation of systems with non-massive primaries, and where both components, with low masses and slower evolutionary rates, can coexist as main-sequence stars. An examination of the observational sample shows that $29 \%$ of the primaries are OB stars; even if this frequency is higher than the frequency of OB stars in the Galaxy, it must be stressed that the sample may carry a bias toward brighter younger stars because it was limited to stars brighter than $m_{V}=7.0$. This strongly suggests that the majority of SBs is composed of low-mass stars, and that a $q_{0}$ limit around 0.1 , allowing simultaneous main sequence components, is the best fit to observations.

Considering the initial mass functions for spectroscopic binaries, Fig. 3 shows that steeper slopes, around 2.35, are not the best fits to observations. Indeed, slopes of about 1.4 seem to be more adequate, even when combined with steeper ones. With respect to $q_{0}$, differences between the three curves at the bottom of Fig. 3 are small, with slight advantage to the homogeneous distribution with slope 1.4 , which has its minimum around $q_{0}=0.08$ to $q_{0}=0.1$, reinforcing the perception gained in the former paragraph. So, a reasonably good fit to observations is obtained from the pairing of stars that are independently formed; the standard IMF is not followed, which suggests an alternative process of binary formation, different from that of single field stars. The process of fragmentation, which is defined as breakup occurring during the dynamical collapse phase of protostellar clouds, leading to equal mass binaries and a dominance of high mass-ratios (Boss 1988), is not confirmed by the present study, because the best fit to observations comes from models with mass ratio distributions favoring low values of $q$. Processes of spectroscopic binary formation, as expected, are different from those of visual binaries.

\section{References}

Abt, H. A., \& Levy, S. G. 1976, ApJS, 30, 273

Abt, H. A., \& Levy, S. G. 1985, ApJS, 59, 229

Batten, A. H., Fletcher, J. M., \& Mann, P. J. 1978, Pub. Dominion Astrophys. Obs. 15,121

Boss, A. P. 1988, Comments Astrophys. 12(4), 169

Dabrowski, J. P., \& Beardsley, W. R. 1977, PASP, 89, 227

Duquennoy, A., \& Mayor, M. 1991, A\&A, 248, 485

Fekel, F. C., Gillies, K., Africano, J., \& Quigley, R. 1988, AJ, 96, 1426

Figer, D. F. 2005, Nature, 434(7030), 192

Fisher, J., Schroder, K. P., \& Smith, R. C. 2005, MNRAS, 361, 495

Garmany, C. D., Conti, P. S., \& Massey, P. C. 1980, AJ, 242, 1063

Giannone, P., \& Giannuzzi, M. A. 1969, Ap\&SS, 84, 292

Goodwin, S. P., \& Kroupa, P. 2005, A\&A, 439, 565

Halbwachs, J. L. 1987, A\&A, 183, 234

Heacox, W. D. 1998, AJ, 115, 325

Hogeveen, S. J. 1990, Ap\&SS, 173, 315

Hogeveen, S. J. 1992a, Ap\&SS, 193, 29

Hogeveen, S. J. 1992b, Ap\&SS, 196, 299

Iben, I. Jr. 1964, ApJ, 140, 1631

Jaschek, C. 1969, Arch. Sc. Geneve, 24(1), 167

Jaschek, C., \& Ferrer, O. 1972, PASP, 84, 292

Kennicut, R. C. 1998, in The Stellar Initial Mass Function, ed. G. Gilmore, \& D. Howell, ASP Conf. Ser., 142, 1

Lang, K. R. 1991, Astrophysical Data: Planets and Stars (Springer), 133

Larson, R. B. 2001, PASP, in The Formation of Binary Stars, , ed. H. Zinnecker, \& R. D. Mathieu (Potsdam), Proc. IAU Symp., 200, 3

Levato, H., Malaroda, S., Morrell, N., \& Solivella, G. 1987, ApJS, 64, 487

Lucy, L. B., \& Ricco, E. 1979, AJ, 84, 401

Pourbaix, D., Tokovinin, A. A., Batten, A. H., et al. 2004, A\&A, 424, 727

Salpeter, E. E. 1955, ApJ, 121, 161

Staniucha, M. 1979, Acta Astron., 29(4), 587

Tohline, J. E., \& Durisen, R. H. 2001, PASP, in The Formation of Binary Stars, ed. H. Zinnecker, \& R. D. Mathieu (Potsdam), Proc. IAU Symp., 200, 40

Tout, C. A. 1991, MNRAS, 250, 701

Trimble, V. 1974, AJ, 79, 967

Trimble, V. 1990, MNRAS, 242, 79

Trimble, V., \& Ostriker, J. P. 1978, A\&A, 63, 437

Warren Jr, W. H., \& Hoffleit, D. 1987, BAAS, 19, 733 
A\&A 525, A26 (2011)

Table 2. Observational data. Column headings: system identification, primary spectral type, primary mass, primary magnitude, system period (days), system orbital excentricity, primary radial velocity $\left(\mathrm{km} \mathrm{s}^{-1}\right)$, mass function, $Y$ value (from Eq. (4)); in last column, a "II" marks SBII systems.

\begin{tabular}{|c|c|c|c|c|c|c|c|c|c|}
\hline Id & $\mathrm{Sp}$ & $m_{1}$ & V & $P$ & $e$ & $k_{1}$ & $f(\mathrm{~m})$ & $Y$ & SB \\
\hline HD 358 & A0 p & 2.9 & 2.17 & 96.70050 & 0.53 & 27.74 & 0.1307179 & $4.507 \mathrm{E}-02$ & II \\
\hline HD 434 & $\mathrm{~A} 4 \mathrm{Vm}$ & 2.2 & 6.52 & 34.26010 & 0.38 & 33.60 & 0.1068118 & $4.855 \mathrm{E}-02$ & \\
\hline HD 861 & A $2 \mathrm{~m}$ & 2.49 & 6.64 & 11.21530 & 0.22 & 43.80 & $9.084 \mathrm{E}-02$ & $3.648 \mathrm{E}-02$ & \\
\hline HD 1273 & G2 V & 0.95 & 6.84 & 411.4490 & 0.57 & 13.90 & $6.365 \mathrm{E}-02$ & $6.700 \mathrm{E}-02$ & \\
\hline HD 3369 & B5 V & 5.9 & 4.35 & 143.6065 & 0.56 & 47.50 & 0.9089235 & 0.1540548 & II \\
\hline HD 3443 & G8 V & 0.85 & 6.31 & 9165.640 & 0.23 & 5.130 & 0.1180137 & 0.1388396 & II \\
\hline HD 3901 & B2 V & 9.8 & 4.80 & 940.2000 & 0.40 & 11.90 & 0.1266737 & $1.292 \mathrm{E}-02$ & \\
\hline HD 4161 & A1 V & 2.7 & 5.63 & 4.467200 & 0.01 & 73.40 & 0.1834277 & $6.793 \mathrm{E}-02$ & II \\
\hline HD 4676 & F8 V & 1.11 & 5.07 & 13.83180 & 0.24 & 57.31 & 0.2473599 & 0.222 & II \\
\hline HD 4727 & B5 V & 5.9 & 4.53 & 4.282700 & 0.03 & 71.70 & 0.1637175 & $2.774 \mathrm{E}-02$ & II \\
\hline HD 4775 & B9.5 V & 3.05 & 5.37 & 2091.200 & 0.53 & 11.02 & 0.1756563 & $5.759 \mathrm{E}-02$ & \\
\hline HD 6118 & B9 V & 3.25 & 5.50 & 81.12000 & 0.90 & 54.30 & 0.1117029 & $3.437 \mathrm{E}-02$ & II \\
\hline HD 7345 & F7 V & 1.21 & 6.27 & 9.075300 & 0.04 & 53.40 & 0.1431687 & 0.1183212 & II \\
\hline HD 8374 & $\mathrm{~F} 2 \mathrm{~m}$ & 1.54 & 5.58 & 35.37100 & 0.63 & 39.00 & 0.1020544 & $6.626 \mathrm{E}-02$ & II \\
\hline HD 9021 & F6 V & 1.24 & 5.82 & 134.0780 & 0.31 & 19.90 & $9.429 \mathrm{E}-02$ & $7.604 \mathrm{E}-02$ & \\
\hline HD 10009 & F7 V & 1.21 & 7.00 & 10540.00 & 0.79 & 9.327 & 0.1950920 & 0.1612331 & II \\
\hline HD 10516 & B2 Vep & 9.8 & 4.07 & 126.6960 & 0.02 & 16.80 & $6.235 \mathrm{E}-02$ & $6.362 \mathrm{E}-03$ & II \\
\hline HD 11291 & B9 p & 3.25 & 5.62 & 5.627000 & 0.02 & 26.50 & $1.086 \mathrm{E}-02$ & $3.344 \mathrm{E}-03$ & \\
\hline HD 11636 & A5 V & 2.0 & 2.60 & 106.9940 & 0.88 & 34.09 & $4.716 \mathrm{E}-02$ & $2.358 \mathrm{E}-02$ & II \\
\hline HD 11753 & A3 V & 2.35 & 5.11 & 41.48900 & 0.32 & 9.000 & $2.671 \mathrm{E}-03$ & $1.136 \mathrm{E}-03$ & \\
\hline HD 11860 & A $0 \mathrm{~V}$ & 2.9 & 6.65 & 7.439100 & 0.00 & 31.60 & $2.437 \mathrm{E}-02$ & $8.406 \mathrm{E}-03$ & II \\
\hline HD 11909 & $\mathrm{~K} 1 \mathrm{p}$ & 0.71 & 5.10 & 1567.660 & 0.36 & 10.80 & 0.1665360 & 0.2345577 & \\
\hline HD 12111 & A4 V & 2.2 & 4.54 & 15011.50 & 0.34 & 4.000 & $8.298 \mathrm{E}-02$ & $3.771 \mathrm{E}-02$ & \\
\hline HD 13974 & G0 V & 1.05 & 4.87 & 10.01950 & 0.01 & 10.52 & $1.211 \mathrm{E}-03$ & $1.153 \mathrm{E}-03$ & \\
\hline HD 14214 & F9 V & 1.09 & 5.56 & 93.50000 & 0.45 & 19.40 & $5.049 \mathrm{E}-02$ & $4.632 \mathrm{E}-02$ & \\
\hline HD 15138 & F4 V & 1.42 & 6.12 & 10.99030 & 0.18 & 46.50 & 0.1092242 & $7.691 \mathrm{E}-02$ & II \\
\hline HD 15814 & F7 V & 1.21 & 6.04 & 19.37870 & 0.39 & 24.00 & $2.172 \mathrm{E}-02$ & $1.795 \mathrm{E}-02$ & II \\
\hline HD 16458 & G8 p... & 0.85 & 5.79 & 2018.000 & 0.10 & 5.830 & $4.091 \mathrm{E}-02$ & $4.813 \mathrm{E}-02$ & \\
\hline HD 16739 & F9 V & 1.09 & 4.92 & 330.9910 & 0.67 & 20.91 & 0.1285 & 0.1179 & II \\
\hline HD 16908 & $\mathrm{~B} 3 \mathrm{~V}$ & 7.6 & 4.66 & 490.0000 & 0.14 & 8.800 & $3.366 \mathrm{E}-02$ & $4.429 \mathrm{E}-03$ & \\
\hline HD 16920 & F2 V & 1.54 & 5.20 & 12.92740 & 0.25 & 58.10 & 0.2390028 & 0.1551966 & II \\
\hline HD 18894 & F8 V & 1.11 & 6.19 & 363.1000 & 0.69 & 24.00 & 0.1976693 & 0.1780804 & II \\
\hline HD 20210 & A9 $\mathrm{m}$ & 1.66 & 6.24 & 5.543500 & 0.03 & 62.70 & 0.1417120 & $8.536 \mathrm{E}-02$ & \\
\hline HD 21242 & G5 V & 0.92 & 6.37 & 6.437870 & 0.00 & 57.86 & 0.1295 & 0.1407 & II \\
\hline HD 21278 & B5 V & 5.9 & 4.98 & 21.65900 & 0.12 & 22.70 & $2.574 \mathrm{E}-02$ & $4.363 \mathrm{E}-03$ & II \\
\hline HD 22203 & B8 V & 3.8 & 4.26 & 6.223600 & 0.20 & 107.0 & 0.7447429 & 0.1959850 & II \\
\hline HD 22805 & A2 V & 2.49 & 6.11 & 20.48700 & 0.61 & 34.20 & $4.234 \mathrm{E}-02$ & $1.700 \mathrm{E}-02$ & \\
\hline HD 23964 & B9.5 V & 3.05 & 6.72 & 16.72500 & 0.00 & 32.40 & $5.907 \mathrm{E}-02$ & $1.936 \mathrm{E}-02$ & II \\
\hline HD 24587 & B5 V & 5.9 & 4.60 & 459.0000 & 0.18 & 21.70 & 0.4635988 & $7.857 \mathrm{E}-02$ & \\
\hline HD 25204 & B3 V & 7.6 & 3.47 & 33.07000 & 0.15 & 10.50 & $3.842 \mathrm{E}-03$ & $5.055 \mathrm{E}-04$ & \\
\hline HD 25823 & $\mathrm{~A} 0 \mathrm{p}$ & 2.9 & 5.19 & 7.227400 & 0.18 & 16.60 & $3.267 \mathrm{E}-03$ & $1.126 \mathrm{E}-03$ & \\
\hline HD 26961 & A2 V & 2.49 & 4.59 & 701.7600 & 0.24 & 11.40 & $9.877 \mathrm{E}-02$ & $3.967 \mathrm{E}-02$ & \\
\hline HD 27176 & A8 V & 1.69 & 5.65 & 4145.720 & 0.17 & 7.355 & 0.1639 & $9.699 \mathrm{E}-02$ & II \\
\hline HD 27295 & B9 Vp & 3.25 & 5.35 & 4.452100 & 0.06 & 9.600 & $4.068 \mathrm{E}-04$ & $1.251 \mathrm{E}-04$ & \\
\hline HD 27376 & B8.5 V & 3.52 & 3.55 & 5.010500 & 0.01 & 63.80 & 0.1351090 & $1.251 \mathrm{E}-04$ & II \\
\hline HD 27991 & F7 V & 1.21 & 6.44 & 2295.090 & 0.71 & 12.36 & 0.1537837 & 0.1270940 & II \\
\hline HD 28271 & F7 V & 1.21 & 6.38 & 460.7000 & 0.31 & 19.35 & 0.2966517 & 0.2451667 & \\
\hline HD 28910 & F0 V & 1.6 & 4.65 & 488.5000 & 0.09 & 18.50 & 0.3173132 & 0.1983207 & \\
\hline HD 30453 & A8 m & 1.69 & 5.86 & 7.050900 & 0.00 & 58.90 & 0.1496 & $8.853 \mathrm{E}-02$ & II \\
\hline HD 30455 & G2 V & 0.95 & 6.79 & 45.43140 & 0.34 & 13.74 & $1.017 \mathrm{E}-02$ & $1.071 \mathrm{E}-02$ & \\
\hline HD 30869 & F5 V & 1.4 & 6.27 & 143.5300 & 0.61 & 18.00 & $4.32 \mathrm{E}-02$ & $3.089 \mathrm{E}-02$ & \\
\hline HD 32537 & F0 V & 1.6 & 4.99 & 391.7000 & 0.37 & 5.800 & $6.364 \mathrm{E}-03$ & $3.977 \mathrm{E}-03$ & \\
\hline HD 32964 & B9.5 V & 3.05 & 5.10 & 5.522700 & 0.10 & 103.8 & 0.6318338 & 0.2071586 & II \\
\hline HD 32990 & B2 V & 9.8 & 5.50 & 58.31000 & 0.19 & 36.70 & 0.2832668 & $2.890 \mathrm{E}-02$ & \\
\hline HD 34364 & B9 V & 3.25 & 6.15 & 4.134600 & 0.00 & 107.2 & 0.5289618 & 0.1627575 & II \\
\hline HD 34759 & B5 V & 5.9 & 5.22 & 35.50000 & 0.00 & 28.00 & $8.092 \mathrm{E}-02$ & $1.371 \mathrm{E}-02$ & \\
\hline HD 34762 & B8 V & 3.8 & 6.33 & 5.433700 & 0.08 & 26.80 & $1.075 \mathrm{E}-02$ & $2.831 \mathrm{E}-03$ & \\
\hline HD 35317 & F7 V & 1.21 & 6.11 & 22.58040 & 0.61 & 53.25 & 0.1761703 & 0.1455953 & II \\
\hline HD 35411 & B1 V & 13.0 & 3.35 & 7.984100 & 0.00 & 145.2 & 2.538238 & 0.1952491 & \\
\hline HD 35411 & B1 V & 13.0 & 3.35 & 3360.230 & 0.10 & 17.50 & 1.842224 & 0.1417095 & \\
\hline HD 36485 & B2 V & 9.8 & 6.85 & 25.59200 & 0.26 & 10.00 & $2.392 \mathrm{E}-03$ & $2.441 \mathrm{E}-04$ & \\
\hline HD 36695 & B1 V & 13.0 & 5.33 & 119.0880 & 0.29 & 13.50 & $2.667 \mathrm{E}-02$ & $2.051 \mathrm{E}-03$ & \\
\hline HD 36964 & B3 V & 7.6 & 6.97 & 4.623900 & 0.12 & 48.10 & $5.228 \mathrm{E}-02$ & $6.879 \mathrm{E}-03$ & \\
\hline HD 37017 & B $1.5 \mathrm{~V}$ & 12.12 & 6.54 & 18.65560 & 0.31 & 36.00 & $7.767 \mathrm{E}-02$ & $6.409 \mathrm{E}-03$ & \\
\hline HD 37041 & $09.5 \mathrm{~V}$ & 18.1 & 5.07 & 20.96433 & 0.23 & 101.3 & 2.0859 & 0.1152 & \\
\hline
\end{tabular}


J. R. Ducati et al.: The mass ratio and initial mass functions in spectroscopic binaries

Table 2. continued.

\begin{tabular}{|c|c|c|c|c|c|c|c|c|c|}
\hline$\overline{\mathrm{Id}}$ & $\mathrm{Sp}$ & $m_{1}$ & $\bar{V}$ & $\overline{\bar{P}}$ & $e$ & $\overline{k_{1}}$ & $\overline{f(m)}$ & $\overline{\bar{Y}}$ & $\overline{\overline{\mathrm{SB}}}$ \\
\hline HD 37438 & $\mathrm{~B} 2 \mathrm{~V}$ & 9.8 & 5.15 & 27.86400 & 0.55 & 25.50 & $2.795 \mathrm{E}-02$ & $2.852 \mathrm{E}-03$ & \\
\hline HD 39357 & B9.5 V & 3.05 & 4.54 & 5.969000 & 0.00 & 48.90 & $7.248 \mathrm{E}-02$ & $2.376 \mathrm{E}-02$ & II \\
\hline HD 39587 & G0 V & 1.05 & 4.41 & 5136.000 & 0.45 & 1.850 & $2.405 \mathrm{E}-03$ & $2.290 \mathrm{E}-03$ & \\
\hline HD 39698 & B2 V & 9.8 & 5.90 & 7.996900 & 0.01 & 70.00 & 0.2848112 & $2.906 \mathrm{E}-02$ & II \\
\hline HD 39780 & A $0 \mathrm{~V}$ & 2.9 & 6.19 & 8.569000 & 0.04 & 62.20 & 0.2136312 & $7.366 \mathrm{E}-02$ & II \\
\hline HD 41335 & B2 Ven & 9.8 & 5.21 & 80.86000 & 0.00 & 9.400 & $6.974 \mathrm{E}-03$ & $7.117 \mathrm{E}-04$ & \\
\hline HD 41357 & A4 m & 2.2 & 5.35 & 28.28000 & 0.56 & 51.40 & 0.2267990 & 0.1030905 & II \\
\hline HD 41511 & B9 V & 3.25 & 4.92 & 260.0000 & 0.13 & 21.00 & 0.2437442 & $7.499 \mathrm{E}-02$ & \\
\hline HD 41753 & B3 V & 7.6 & 4.40 & 131.2110 & 0.64 & 33.30 & 0.2282595 & $3.003 \mathrm{E}-02$ & \\
\hline HD 42083 & A $2 \mathrm{~m}$ & 2.49 & 6.17 & 106.0000 & 0.63 & 40.50 & 0.3425007 & 0.1375505 & II \\
\hline HD 44402 & B3 V & 7.6 & 3.02 & 675.0000 & 0.57 & 13.50 & $9.566 \mathrm{E}-02$ & $1.258 \mathrm{E}-02$ & \\
\hline HD 44691 & A $3 \mathrm{~m}$ & 2.35 & 5.50 & 9.945100 & 0.08 & 65.65 & 0.2900876 & 0.1234415 & II \\
\hline HD 45088 & K3 V & 0.68 & 6.79 & 6.991900 & 0.15 & 56.60 & 0.1272407 & 0.1871187 & II \\
\hline HD 47839 & $\mathrm{O} 7 \mathrm{Ve}$ & 26.5 & 4.66 & 9247.000 & 0.67 & 9.400 & 0.3263146 & $1.231 \mathrm{E}-02$ & \\
\hline HD 48766 & F6 V & 1.24 & 6.28 & 4.258560 & 0.00 & 12.82 & $9.318 \mathrm{E}-04$ & $7.514 \mathrm{E}-04$ & \\
\hline HD 48915 & A1 V & 2.7 & 1.47 & 18276.70 & 0.59 & 2.400 & $1.381 \mathrm{E}-02$ & $5.115 \mathrm{E}-03$ & \\
\hline HD 51424 & A2 V & 2.49 & 6.34 & 6007.000 & 0.13 & 3.500 & $2.598 \mathrm{E}-02$ & $1.043 \mathrm{E}-02$ & \\
\hline HD 54563 & G8 V & 0.85 & 6.43 & 113.3460 & 0.40 & 20.80 & $8.154 \mathrm{E}-02$ & $9.594 \mathrm{E}-02$ & \\
\hline HD 58661 & B9 pHgMn & 3.25 & 5.72 & 1834.000 & 0.30 & 5.000 & $2.066 \mathrm{E}-02$ & $6.359 \mathrm{E}-03$ & \\
\hline HD 59543 & B2 V & 9.8 & 6.94 & 17.91100 & 0.52 & 45.60 & 0.10099127 & $1.121 \mathrm{E}-02$ & \\
\hline HD 60179 & A1 V & 2.7 & 1.58 & 9.212800 & 0.50 & 12.90 & $1.334 \mathrm{E}-03$ & $4.940 \mathrm{E}-04$ & \\
\hline HD 61859 & F7 V & 1.21 & 5.97 & 31.50000 & 0.21 & 45.20 & 0.2823261 & 0.2333274 & II \\
\hline HD 64096 & G1 V & 0.99 & 5.16 & 8291.550 & 0.74 & 9.125 & 0.1990 & 0.2010 & II \\
\hline HD 66824 & A1 V & 2.7 & 6.36 & 18.72200 & 0.17 & 64.60 & 0.5015938 & 0.1857755 & II \\
\hline HD 68256 & G5 V & 0.92 & 6.20 & 6302.000 & 0.11 & 4.280 & $5.022 \mathrm{E}-02$ & $5.459 \mathrm{E}-02$ & \\
\hline HD 68351 & B9 p & 3.25 & 5.64 & 635.7300 & 0.55 & 9.600 & $3.402 \mathrm{E}-02$ & $1.046 \mathrm{E}-02$ & \\
\hline HD 68520 & B5 V & 5.9 & 4.34 & 14.16830 & 0.00 & 66.70 & 0.4366181 & $7.400 \mathrm{E}-02$ & \\
\hline HD 71581 & A1 V & 2.7 & 6.56 & 4.596200 & 0.10 & 103.6 & 0.5228022 & 0.1936305 & II \\
\hline HD 71663 & A $5 \mathrm{~m}$ & 2.0 & 6.38 & 5.977100 & 0.00 & 42.10 & $4.631 \mathrm{E}-02$ & $2.315 \mathrm{E}-02$ & II \\
\hline HD 72208 & B9 pHg: & 3.25 & 6.83 & 22.01160 & 0.38 & 48.20 & 0.2025841 & $6.233 \mathrm{E}-02$ & \\
\hline HD 73712 & A9 Vn & 1.66 & 6.78 & 48.71700 & 0.11 & 31.60 & 0.1567537 & $9.442 \mathrm{E}-02$ & II \\
\hline HD 73731 & A5 m & 2.0 & 6.30 & 35.20200 & 0.32 & 53.00 & 0.4628345 & 0.2314172 & II \\
\hline HD 75759 & B0 V & 17.5 & 5.98 & 33.31100 & 0.63 & 121.3 & 2.891749 & 0.1652428 & II \\
\hline HD 75767 & G1 V & 0.99 & 6.57 & 10.24806 & 0.00 & 23.59 & $1.397 \mathrm{E}-02$ & $1.411 \mathrm{E}-02$ & \\
\hline HD 76370 & A $2 \mathrm{~m}$ & 2.49 & 6.07 & 18.83020 & 0.49 & 13.73 & $3.333 \mathrm{E}-03$ & $1.338 \mathrm{E}-03$ & \\
\hline HD 76644 & A7 V & 1.79 & 3.14 & 4028.000 & 0.36 & 6.000 & 7.337E-02 & $4.098 \mathrm{E}-02$ & \\
\hline HD 76943 & F5 V & 1.4 & 3.97 & 7980.700 & 0.15 & 4.000 & $5.126 \mathrm{E}-02$ & $3.661 \mathrm{E}-02$ & \\
\hline HD 77350 & B9 p & 3.25 & 5.43 & 1401.400 & 0.35 & 7.700 & $5.461 \mathrm{E}-02$ & $1.680 \mathrm{E}-02$ & \\
\hline HD 77464 & B2.5 V & 9.5 & 6.69 & 6.889500 & 0.00 & 127.0 & 1.465566 & 0.1542701 & II \\
\hline HD 78316 & B8 p & 3.8 & 5.24 & 6.393300 & 0.13 & 67.40 & 0.1981563 & $5.214 \mathrm{E}-02$ & \\
\hline HD 79028 & F9 V & 1.09 & 5.17 & 16.23970 & 0.009 & 35.30 & $7.328 \mathrm{E}-02$ & $6.723 \mathrm{E}-02$ & \\
\hline HD 79096 & G9 V & 0.82 & 6.51 & 988.0580 & 0.43 & 11.49 & 0.1145 & 0.1396 & II \\
\hline HD 79193 & A $3 \mathrm{~m}$ & 2.35 & 6.11 & 7.750500 & 0.09 & 70.10 & 0.2739000 & 0.1165532 & II \\
\hline HD 79763 & A1 V & 2.7 & 5.97 & 15.98600 & 0.50 & 63.30 & 0.2734949 & 0.1012944 & II \\
\hline HD 81809 & G0 V & 1.05 & 5.74 & 12589.60 & 0.24 & 4.752 & 0.1277253 & 0.1216432 & II \\
\hline HD 81858 & F8 V & 1.11 & 5.40 & 42678.50 & 0.56 & 2.200 & $2.683 \mathrm{E}-02$ & $2.417 \mathrm{E}-02$ & \\
\hline HD 83809 & A5 V & 2.0 & 3.52 & 14.49808 & 0.00 & 54.80 & 0.2477 & 0.1238 & II \\
\hline HD 86118 & B2 V & 9.8 & 6.64 & 4.478000 & 0.28 & 167.0 & 1.916257 & 0.1955364 & II \\
\hline HD 86146 & F5 V & 1.4 & 5.12 & 9.283500 & 0.00 & 18.90 & $6.508 \mathrm{E}-03$ & $4.649 \mathrm{E}-03$ & II \\
\hline HD 87810 & F3 V & 1.47 & 6.67 & 12.94724 & 0.43 & 55.50 & 0.1667240 & 0.1134177 & II \\
\hline HD 88215 & F5 V & 1.4 & 5.30 & 28.09800 & 0.07 & 10.10 & $2.984 \mathrm{E}-03$ & $2.131 \mathrm{E}-03$ & \\
\hline HD 89822 & A0 p & 2.9 & 4.93 & 11.57910 & 0.26 & 38.90 & $6.372 \mathrm{E}-02$ & $2.197 \mathrm{E}-02$ & II \\
\hline HD 92168 & F8 V & 1.11 & 5.85 & 7.799100 & 0.02 & 24.10 & $1.133 \mathrm{E}-02$ & $1.020 \mathrm{E}-02$ & \\
\hline HD 93903 & A $3 \mathrm{~m}$ & 2.35 & 5.79 & 6.166900 & 0.00 & 46.60 & $6.480 \mathrm{E}-02$ & $2.757 \mathrm{E}-02$ & \\
\hline HD 94334 & A1 V & 2.7 & 4.68 & 15.83070 & 0.31 & 22.20 & $1.545 \mathrm{E}-02$ & $5.725 \mathrm{E}-03$ & \\
\hline HD 96528 & A5 m & 2.0 & 6.46 & 40.45000 & 0.10 & 18.00 & $2.413 \mathrm{E}-02$ & $1.206 \mathrm{E}-02$ & \\
\hline HD 98088 & A3 Vp & 2.35 & 6.14 & 5.905100 & 0.17 & 74.70 & 0.2446197 & 0.1040935 & II \\
\hline HD 98231 & G5 V & 0.92 & 4.41 & 670.2400 & 0.53 & 8.950 & $3.042 \mathrm{E}-02$ & $3.307 \mathrm{E}-02$ & \\
\hline HD 99967 & K0 V & 0.79 & 6.38 & 74.86100 & 0.03 & 28.80 & 0.1854612 & 0.2347610 & \\
\hline HD 101013 & $\mathrm{~K} 0 \mathrm{pBa} 3$ & 0.79 & 6.14 & 1710.900 & 0.19 & 6.070 & $3.760 \mathrm{E}-02$ & $4.760 \mathrm{E}-02$ & \\
\hline HD 101606 & F5 V & 1.4 & 5.74 & 267.5078 & 0.85 & 39.07 & 0.2364242 & 0.1688744 & II \\
\hline HD 102509 & A7 V & 1.79 & 4.53 & 71.69060 & 0.00 & 30.12 & 0.2034 & 0.1136 & II \\
\hline HD 103578 & A3 V & 2.35 & 5.50 & 6.625400 & 0.02 & 57.60 & 0.1314091 & $5.591 \mathrm{E}-02$ & \\
\hline HD 104321 & A4 V & 2.2 & 4.64 & 282.6900 & 0.27 & 26.20 & 0.4713145 & 0.2142338 & \\
\hline HD 105981 & $\mathrm{~K} 2 \mathrm{~V}$ & 0.69 & 5.66 & 461.0000 & 0.17 & 14.30 & 0.1339712 & 0.1941611 & \\
\hline HD 106516 & F5 V & 1.4 & 6.11 & 843.9000 & 0.05 & 7.930 & $4.353 \mathrm{E}-02$ & $3.109 \mathrm{E}-02$ & \\
\hline HD 107259 & A2 V & 2.49 & 3.88 & 71.79190 & 0.27 & 26.67 & 0.1262 & $5.070 \mathrm{E}-02$ & II \\
\hline
\end{tabular}


Table 2. continued.

\begin{tabular}{|c|c|c|c|c|c|c|c|c|c|}
\hline Id & $\overline{S p}$ & $m_{1}$ & $\bar{V}$ & $\overline{\bar{P}}$ & $e$ & $\overline{k_{1}}$ & $\overline{f(m)}$ & $\overline{\bar{Y}}$ & $\overline{\overline{\mathrm{SB}}}$ \\
\hline HD 107259 & A2 V & 2.49 & 3.88 & 4791.900 & 0.08 & 4.820 & $5.519 \mathrm{E}-02$ & $2.216 \mathrm{E}-02$ & \\
\hline HD 107935 & A7 V & 1.79 & 6.71 & 176.0800 & 0.19 & 19.40 & 0.1263484 & $7.058 \mathrm{E}-02$ & \\
\hline HD 108642 & A $2 \mathrm{~m}$ & 2.49 & 6.52 & 11.78243 & 0.00 & 41.14 & $8.519 \mathrm{E}-02$ & $3.412 \mathrm{E}-02$ & \\
\hline HD 108945 & A2 pvar & 2.49 & 5.44 & 18.81300 & 0.19 & 30.50 & $5.245 \mathrm{E}-02$ & $2.106 \mathrm{E}-02$ & II \\
\hline HD 110854 & $\mathrm{~A} 0 \mathrm{~V}$ & 2.9 & 6.56 & 7.904000 & 0.03 & 69.60 & 0.2763723 & $9.530 \mathrm{E}-02$ & II \\
\hline HD 112486 & A $5 \mathrm{~m}$ & 2.0 & 5.82 & 5.125900 & 0.00 & 65.80 & 0.1516542 & $7.582 \mathrm{E}-02$ & II \\
\hline HD 114911 & B8 V & 3.8 & 4.79 & 20.00520 & 0.12 & 56.50 & 0.3666450 & $9.648 \mathrm{E}-02$ & \\
\hline HD 116656 & A2 V & 2.49 & 2.27 & 20.53850 & 0.53 & 67.26 & 0.3957 & 0.1589 & II \\
\hline HD 116658 & B1 V & 13.0 & 0.97 & 4.014500 & 0.18 & 120.0 & 0.6856863 & $5.274 \mathrm{E}-02$ & II \\
\hline HD 120710 & B8 V & 3.8 & 6.06 & 17.42800 & 0.21 & 17.00 & $8.310 \mathrm{E}-03$ & $2.186 \mathrm{E}-03$ & \\
\hline HD 121648 & F2 V & 1.54 & 6.79 & 4.991670 & 0.00 & 89.85 & 0.3760 & 0.2441 & II \\
\hline HD 122742 & G8 V & 0.85 & 6.36 & 3617.000 & 0.48 & 6.410 & $6.079 \mathrm{E}-02$ & $7.857 \mathrm{E}-02$ & \\
\hline HD 125248 & A0 p & 2.9 & 5.89 & 1607.070 & 0.21 & 7.500 & $6.580 \mathrm{E}-02$ & $2.269 \mathrm{E}-02$ & \\
\hline HD 126983 & A2 V & 2.49 & 5.36 & 11.82000 & 0.33 & 76.60 & 0.4640875 & 0.1863805 & II \\
\hline HD 128620 & G2 V & 0.95 & 0.01 & 29188.10 & 0.52 & 4.610 & 0.1850 & 0.1948 & II \\
\hline HD 129132 & G0 V & 1.05 & 6.76 & 101.6060 & 0.12 & 19.00 & $7.081 \mathrm{E}-02$ & $6.744 \mathrm{E}-02$ & \\
\hline HD 129132 & G0 V & 1.05 & 6.76 & 3385.000 & 0.07 & 8.500 & 0.2242 & 0.2040 & \\
\hline HD 131511 & K1 V & 0.71 & 6.00 & 125.3960 & 0.51 & 19.10 & $5.774 \mathrm{E}-02$ & $8.133 \mathrm{E}-02$ & \\
\hline HD 137052 & F5 V & 1.4 & 4.93 & 226.9500 & 0.68 & 14.00 & $2.549 \mathrm{E}-02$ & $1.820 \mathrm{E}-02$ & \\
\hline HD 137108 & G0 V & 1.05 & 4.98 & 15189.00 & 0.27 & 4.709 & 0.1470 & 0.1400 & II \\
\hline HD 137763 & K1 V & 0.71 & 6.83 & 889.6200 & 0.97 & 37.14 & $5.131 \mathrm{E}-02$ & $7.227 \mathrm{E}-02$ & II \\
\hline HD 137909 & F0 p & 1.6 & 3.66 & 3833.580 & 0.41 & 9.200 & 0.2352238 & 0.1470149 & \\
\hline HD 138213 & A $5 \mathrm{~m}$ & 2.0 & 6.15 & 105.9500 & 0.00 & 10.80 & $1.386 \mathrm{E}-02$ & $6.930 \mathrm{E}-03$ & \\
\hline HD 139461 & F6 V & 1.24 & 6.48 & 887.6600 & 0.90 & 13.96 & $2.077 \mathrm{E}-02$ & $1.675 \mathrm{E}-02$ & \\
\hline HD 140008 & B6 V & 5.0 & 4.74 & 12.26000 & 0.19 & 63.30 & 0.3056019 & $6.112 \mathrm{E}-02$ & II \\
\hline HD 141458 & A0 V & 2.9 & 6.82 & 28.94900 & 0.64 & 60.60 & 0.3035131 & 0.1046597 & II \\
\hline HD 142096 & B2.5 V & 9.5 & 5.03 & 12.46190 & 0.40 & 33.50 & $3.745 \mathrm{E}-02$ & $3.942 \mathrm{E}-03$ & \\
\hline HD 142883 & B3 V & 7.6 & 5.85 & 10.53500 & 0.58 & 64.00 & 0.1479528 & $1.946 \mathrm{E}-02$ & \\
\hline HD 143807 & A0 $\mathrm{pHg}$ & 2.9 & 5.12 & 35.47400 & 0.56 & 2.300 & $2.548 \mathrm{E}-05$ & $8.789 \mathrm{E}-06$ & \\
\hline HD 144426 & A $3 \mathrm{~m}$ & 2.35 & 6.28 & 8.855000 & 0.38 & 31.60 & $2.296 \mathrm{E}-02$ & $9.772 \mathrm{E}-03$ & \\
\hline HD 145389 & B9 p & 3.25 & 4.24 & 560.5000 & 0.47 & 2.400 & $5.533 \mathrm{E}-04$ & $1.702 \mathrm{E}-04$ & \\
\hline HD 145482 & B2 V & 9.8 & 4.59 & 5.780500 & 0.19 & 31.50 & $1.775 \mathrm{E}-02$ & $1.811 \mathrm{E}-03$ & \\
\hline HD 147584 & G0 V & 1.05 & 4.90 & 12.97620 & 0.06 & 7.400 & $5.431 \mathrm{E}-04$ & $5.172 \mathrm{E}-04$ & \\
\hline HD 147869 & A2 p(Sr) & 2.49 & 5.84 & 5.019928 & 0.21 & 14.83 & $1.589 \mathrm{E}-03$ & $6.381 \mathrm{E}-04$ & \\
\hline HD 149632 & A2 V & 2.49 & 6.41 & 10.56000 & 0.43 & 62.40 & 0.1960833 & $7.874 \mathrm{E}-02$ & II \\
\hline HD 151613 & F2 V & 1.54 & 4.85 & 363.5700 & 0.35 & 6.000 & $6.703 \mathrm{E}-03$ & $4.353 \mathrm{E}-03$ & \\
\hline HD 152830 & F3 Vs & 1.47 & 6.34 & 11.85859 & 0.36 & 27.44 & $2.066 \mathrm{E}-02$ & $1.405 \mathrm{E}-02$ & \\
\hline HD 153597 & F6 V & 1.24 & 4.88 & 52.10890 & 0.21 & 17.60 & $2.757 \mathrm{E}-02$ & $2.223 \mathrm{E}-02$ & \\
\hline HD 153808 & A0 V & 2.9 & 3.92 & 4.023500 & 0.02 & 70.70 & 0.1475734 & $5.088 \mathrm{E}-02$ & II \\
\hline HD 154905 & F7 V & 1.21 & 5.83 & 2270.000 & 0.43 & 2.800 & $3.808 \mathrm{E}-03$ & $3.147 \mathrm{E}-03$ & \\
\hline HD 155375 & A1 m & 2.7 & 6.60 & 23.24500 & 0.43 & 27.70 & $3.775 \mathrm{E}-02$ & $1.398 \mathrm{E}-02$ & \\
\hline HD 157482 & F9 Vn & 1.09 & 5.56 & 2018.800 & 0.67 & 12.89 & 0.1823576 & 0.1673005 & II \\
\hline HD 157950 & F3 V & 1.47 & 4.53 & 26.27650 & 0.49 & 47.50 & 0.1937267 & 0.1317869 & II \\
\hline HD 158261 & A0 V & 2.9 & 5.94 & 5.918200 & 0.03 & 25.10 & $9.705 \mathrm{E}-03$ & $3.346 \mathrm{E}-03$ & \\
\hline HD 159082 & B9.5 V & 3.05 & 6.42 & 6.797500 & 0.07 & 48.90 & $8.193 \mathrm{E}-02$ & $2.686 \mathrm{E}-02$ & \\
\hline HD 159560 & A4 m & 2.2 & 4.89 & 38.03400 & 0.03 & 10.00 & $3.944 \mathrm{E}-03$ & $1.792 \mathrm{E}-03$ & \\
\hline HD 160346 & K3 V & 0.68 & 6.52 & 83.72800 & 0.22 & 5.700 & $1.488 \mathrm{E}-03$ & $2.188 \mathrm{E}-03$ & \\
\hline HD 160922 & F5 V & 1.4 & 4.80 & 5.279800 & 0.00 & 35.80 & $2.515 \mathrm{E}-02$ & $1.796 \mathrm{E}-02$ & II \\
\hline HD 161573 & B4 V & 6.86 & 6.85 & 19.08500 & 0.14 & 5.800 & $3.753 \mathrm{E}-04$ & $5.472 \mathrm{E}-05$ & \\
\hline HD 162515 & B9.5 V & 3.05 & 6.51 & 6.678300 & 0.02 & 57.60 & 0.1324583 & $4.342 \mathrm{E}-02$ & \\
\hline HD 162780 & B9 V & 3.25 & 6.89 & 6.622600 & 0.54 & 13.20 & $9.431 \mathrm{E}-04$ & $2.901 \mathrm{E}-04$ & \\
\hline HD 163840 & G0 V & 1.05 & 6.39 & 881.8080 & 0.41 & 11.22 & $9.807 \mathrm{E}-02$ & $9.340 \mathrm{E}-02$ & II \\
\hline HD 165341 & K0 V & 0.79 & 4.02 & 32280.50 & 0.50 & 3.657 & 0.1064 & 0.1347 & II \\
\hline HD 166285 & F5 V & 1.4 & 5.68 & 199.5500 & 0.30 & 14.20 & $5.140 \mathrm{E}-02$ & $3.671 \mathrm{E}-02$ & II \\
\hline HD 166865 & K2 V & 0.69 & 6.04 & 10.52785 & 0.37 & 39.14 & $5.256 \mathrm{E}-02$ & $7.618 \mathrm{E}-02$ & II \\
\hline HD 166866 & F7 V & 1.21 & 5.68 & 1247.200 & 0.97 & 44.79 & 0.1246728 & 0.1030354 & II \\
\hline HD 167858 & F1 V & 1.57 & 6.62 & 4.485180 & 0.00 & 6.200 & $1.110 \mathrm{E}-04$ & $4.070 \mathrm{E}-05$ & \\
\hline HD 167954 & F8 V & 1.11 & 6.85 & 120.0074 & 0.04 & 15.50 & $4.629 \mathrm{E}-02$ & $4.171 \mathrm{E}-02$ & \\
\hline HD 168913 & A $5 \mathrm{~m}$ & 2.0 & 5.63 & 5.127000 & 0.00 & 70.10 & 0.1972082 & $9.860 \mathrm{E}-02$ & II \\
\hline HD 169981 & A2 V & 2.49 & 5.88 & 9.612000 & 0.47 & 28.50 & $1.589 \mathrm{E}-02$ & $6.381 \mathrm{E}-03$ & \\
\hline HD 170000 & A0 $p$ & 2.9 & 4.18 & 26.76800 & 0.39 & 26.60 & $4.084 \mathrm{E}-02$ & $1.408 \mathrm{E}-02$ & \\
\hline HD 170153 & F7 V & 1.21 & 3.57 & 280.5170 & 0.41 & 17.31 & 0.1146 & $9.474 \mathrm{E}-02$ & II \\
\hline HD 171978 & A2 V & 2.49 & 5.77 & 14.67400 & 0.21 & 38.60 & $8.190 \mathrm{E}-02$ & $3.289 \mathrm{E}-02$ & II \\
\hline HD 172103 & F4 V & 1.42 & 6.65 & 39.52600 & 0.37 & 21.68 & $3.345 \mathrm{E}-02$ & $2.355 \mathrm{E}-02$ & II \\
\hline HD 173282 & F5 V & 1.4 & 6.36 & 33.16070 & 0.72 & 49.80 & 0.1421 & 0.1015 & II \\
\hline HD 173654 & A2 Vm & 2.49 & 5.90 & 4.765300 & 0.02 & 17.30 & $2.560 \mathrm{E}-03$ & $1.028 \mathrm{E}-03$ & II \\
\hline HD 174933 & B7 V & 4.4 & 5.48 & 6.362400 & 0.12 & 17.70 & $3.585 \mathrm{E}-03$ & $8.147 \mathrm{E}-04$ & \\
\hline
\end{tabular}


J. R. Ducati et al.: The mass ratio and initial mass functions in spectroscopic binaries

Table 2. continued.

\begin{tabular}{|c|c|c|c|c|c|c|c|c|c|}
\hline$\overline{I d}$ & $\mathrm{Sp}$ & $m_{1}$ & $\bar{V}$ & $\overline{\bar{P}}$ & $e$ & $\overline{k_{1}}$ & $\overline{f(m)}$ & $\overline{\bar{Y}}$ & $\overline{\overline{\mathrm{SB}}}$ \\
\hline HD 175426 & $\mathrm{~B} 2.5 \mathrm{~V}$ & 9.5 & 5.75 & 88.35200 & 0.37 & 39.70 & 0.4603478 & $4.845 \mathrm{E}-02$ & \\
\hline HD 177863 & B8 V & 3.8 & 6.29 & 11.91540 & 0.60 & 42.50 & $4.822 \mathrm{E}-02$ & $1.269 \mathrm{E}-02$ & \\
\hline HD 178322 & B6 V & 5.0 & 5.87 & 12.47000 & 0.05 & 79.70 & 0.6531565 & 0.1306313 & II \\
\hline HD 178428 & G5 V & 0.92 & 6.08 & 21.95536 & 0.08 & 13.42 & $5.457 \mathrm{E}-03$ & $5.932 \mathrm{E}-03$ & \\
\hline HD 178449 & F0 V & 1.6 & 5.23 & 42.85700 & 0.00 & 13.10 & $1.000 \mathrm{E}-02$ & $6.253 \mathrm{E}-03$ & \\
\hline HD 178911 & G1 V & 0.99 & 6.90 & 1296.300 & 0.58 & 6.570 & $2.014 \mathrm{E}-02$ & $2.035 \mathrm{E}-02$ & II \\
\hline HD 180939 & B5 V & 5.9 & 6.79 & 4.477300 & 0.00 & 55.00 & $7.735 \mathrm{E}-02$ & $1.311 \mathrm{E}-02$ & II \\
\hline HD 183007 & A3 $p$ & 2.35 & 5.69 & 164.6400 & 0.12 & 11.80 & $2.748 \mathrm{E}-02$ & $1.169 \mathrm{E}-02$ & \\
\hline HD 183056 & B8 p & 3.8 & 5.11 & 35.02250 & 0.45 & 5.700 & $4.797 \mathrm{E}-04$ & $1.262 \mathrm{E}-04$ & \\
\hline HD 184467 & K1 V & 0.71 & 6.59 & 494.0910 & 0.36 & 9.564 & $3.645 \mathrm{E}-$ & $5.133 \mathrm{E}-02$ & II \\
\hline HD 184552 & A8 $\mathrm{m}$ & 1.69 & 5.66 & 8.115800 & 0.14 & 20.90 & $7.469 \mathrm{E}-03$ & $4.419 \mathrm{E}-03$ & \\
\hline HD 185912 & F5 V & 1.4 & 5.86 & 7.640800 & 0.54 & 88.20 & 0.3246152 & 0.2318680 & II \\
\hline HD 188164 & A $3 p$ & 2.35 & 6.38 & 14.98590 & 0.56 & 42.90 & $6.987 \mathrm{E}-02$ & $2.973 \mathrm{E}-02$ & II \\
\hline HD 189178 & B5 Vp & 5.9 & 5.41 & 70.23000 & 0.34 & 43.50 & 0.4993 & $8.462 \mathrm{E}-02$ & \\
\hline HD 189340 & F8 V & 1.11 & 6.21 & 1786.270 & 0.59 & 4.688 & $9.988 \mathrm{E}-03$ & $8.998 \mathrm{E}-03$ & II \\
\hline HD 189783 & F5 V & 1.4 & 6.98 & 4.469600 & 0.10 & 41.30 & $3.220 \mathrm{E}-02$ & $2.300 \mathrm{E}-02$ & \\
\hline HD 190229 & B9 p & 3.25 & 5.67 & 61.54100 & 0.49 & 4.200 & $3.136 \mathrm{E}-04$ & $9.650 \mathrm{E}-05$ & \\
\hline HD 192276 & B7 V & 4.4 & 6.92 & 7.185800 & 0.01 & 18.50 & $4.724 \mathrm{E}-03$ & $1.073 \mathrm{E}-03$ & \\
\hline HD 193495 & B8 V & 3.8 & 3.08 & 8.677700 & 0.34 & 35.10 & $3.241 \mathrm{E}-02$ & $8.529 \mathrm{E}-03$ & \\
\hline HD 193964 & B9 V & 3.25 & 5.62 & 5.298100 & 0.04 & 49.70 & $6.738 \mathrm{E}-02$ & $2.073 \mathrm{E}-02$ & \\
\hline HD 194215 & K3 V & 0.68 & 5.84 & 377.6000 & 0.07 & 11.20 & $5.468 \mathrm{E}-02$ & $8.042 \mathrm{E}-02$ & \\
\hline HD 196133 & A0 $p$ & 2.9 & 6.70 & 87.68700 & 0.76 & 32.50 & $8.581 \mathrm{E}-02$ & $2.959 \mathrm{E}-02$ & \\
\hline HD 196544 & A2 V & 2.49 & 5.44 & 11.03900 & 0.23 & 26.00 & $1.857 \mathrm{E}-02$ & $7.458 \mathrm{E}-03$ & \\
\hline HD 198391 & A1 Vs & 2.7 & 6.33 & 10.88300 & 0.39 & 31.40 & $2.731 \mathrm{E}-02$ & $1.011 \mathrm{E}-02$ & \\
\hline HD 201433 & B9 V & 3.25 & 5.55 & 154.0900 & 0.00 & 9.700 & $1.460 \mathrm{E}-02$ & $4.493 \mathrm{E}-03$ & \\
\hline HD 202275 & F5 V & 1.4 & 4.49 & 2082.100 & 0.46 & 12.40 & 0.2885978 & 0.2061413 & II \\
\hline HD 202940 & G5 V & 0.92 & 6.55 & 21.34620 & 0.25 & 24.00 & $2.781 \mathrm{E}-02$ & $3.023 \mathrm{E}-02$ & \\
\hline HD 203064 & $\mathrm{O} 7 \mathrm{~V}(\mathrm{f})$ & 26.5 & 5.00 & 5.100000 & 0.00 & 30.00 & $1.430 \mathrm{E}-02$ & $5.396 \mathrm{E}-04$ & \\
\hline HD 203439 & A2 V & 2.49 & 6.04 & 20.30000 & 0.44 & 45.70 & 0.1457058 & $5.851 \mathrm{E}-02$ & II \\
\hline HD 203858 & A2 V & 2.49 & 6.16 & 6.946300 & 0.00 & 71.40 & 0.2625758 & 0.1054521 & II \\
\hline HD 204188 & A8 $\mathrm{m}$ & 1.69 & 6.08 & 21.72200 & 0.00 & 46.00 & 0.2195 & 0.1299 & \\
\hline HD 206155 & A $3 \mathrm{~m}$ & 2.35 & 6.93 & 1464.000 & 0.52 & 4.400 & $8.071 \mathrm{E}-03$ & $3.434 \mathrm{E}-03$ & II \\
\hline HD 206672 & B3 V & 7.6 & 4.67 & 26.33000 & 0.00 & 16.50 & $1.228 \mathrm{E}-02$ & $1.616 \mathrm{E}-03$ & \\
\hline HD 207650 & A0 V & 2.9 & 5.09 & 5.304700 & 0.53 & 37.00 & $1.701 \mathrm{E}-02$ & $5.867 \mathrm{E}-03$ & II \\
\hline HD 208132 & A1 m & 2.7 & 6.37 & 8.303440 & 0.19 & 21.80 & $8.434 \mathrm{E}-03$ & $3.123 \mathrm{E}-03$ & \\
\hline HD 208509 & A2 V & 2.49 & 6.64 & 28.81840 & 0.12 & 17.10 & $1.461 \mathrm{E}-02$ & $5.869 \mathrm{E}-03$ & \\
\hline HD 208776 & G0 V & 1.05 & 6.94 & 2624.000 & 0.27 & 5.460 & $3.959 \mathrm{E}-02$ & $3.770 \mathrm{E}-02$ & \\
\hline HD 210027 & F5 V & 1.4 & 3.76 & 10.21300 & 0.00 & 48.10 & 0.1180307 & $8.430 \mathrm{E}-02$ & II \\
\hline HD 213429 & F7 V & 1.21 & 6.26 & 630.1400 & 0.38 & 11.44 & $7.734 \mathrm{E}-02$ & $6.392 \mathrm{E}-02$ & II \\
\hline HD 214608 & F9 V & 1.09 & 6.83 & 551.6000 & 0.05 & 13.10 & 0.1282963 & 0.1177030 & II \\
\hline HD 214608 & F9 V & 1.09 & 6.83 & 10957.30 & 0.30 & 5.900 & 0.2028748 & 0.1861237 & II \\
\hline HD 214686 & F7 V & 1.21 & 6.89 & 21.70140 & 0.41 & 55.10 & 0.2860591 & 0.2364125 & II \\
\hline HD 216608 & A $3 \mathrm{~m}$ & 2.35 & 5.81 & 24.22800 & 0.20 & 5.800 & $4.617 \mathrm{E}-04$ & $1.964 \mathrm{E}-04$ & \\
\hline HD 217792 & F0 V & 1.6 & 5.10 & 178.3177 & 0.53 & 21.30 & 0.1091249 & $8.820 \mathrm{E}-02$ & \\
\hline HD 219749 & B9 pSi & 3.25 & 6.48 & 48.30400 & 0.50 & 25.70 & $5.530 \mathrm{E}-02$ & $1.701 \mathrm{E}-02$ & \\
\hline HD 221253 & B3 V & 7.6 & 4.88 & 6.066300 & 0.25 & 56.70 & 0.1042405 & $1.371 \mathrm{E}-02$ & \\
\hline HD 221950 & F6 V & 1.24 & 5.66 & 45.45900 & 0.37 & 40.10 & 0.2440906 & 0.1968473 & II \\
\hline HD 222098 & A2 V & 2.49 & 6.26 & 11.22980 & 0.04 & 26.70 & $2.214 \mathrm{E}-02$ & $8.893 \mathrm{E}-03$ & \\
\hline HD 223778 & K3 V & 0.68 & 6.40 & 7.753100 & 0.00 & 39.90 & $5.114 \mathrm{E}-02$ & $7.521 \mathrm{E}-02$ & II \\
\hline HD 224930 & G2 V & 0.95 & 5.75 & 9610.000 & 0.37 & 4.490 & $7.243 \mathrm{E}-02$ & $7.642 \mathrm{E}-02$ & \\
\hline
\end{tabular}

